\title{
Recubrimiento directo con agregado trióxido mineral (MTA) comparado con hidróxido de calcio para caries dentinaria profunda en pacientes con dentición permanente.
}

\section{Direct pulp capping using mineral trioxide aggregate (MTA) versus calcium hydroxide for deep dental caries in permanent dentition.}

\author{
Loretto Díaz $^{1,2}$, Gabriela Flores ${ }^{1,2}$, Ana María Palma ${ }^{1,2^{*}}$
}

\author{
1. Escuela de Odontología, Facultad de Medicina, \\ Pontificia Universidad Católica de Chile, Santiago, \\ Chile \\ 2. Proyecto Epistemonikos, Santiago, Chile \\ * Correspondencia Autor: Ana María Palma \\ | Dirección: Centro Evidencia UC, Pontificia \\ Universidad Católica de Chile, Diagonal Paraguay \\ 476, Santiago, Chile | E-mail: apalmay@uc.cl
}

\begin{abstract}
RESUMEN
Introducción: El recubrimiento pulpar directo es un método para tratar la pulpa vital expuesta conservando su vitalidad. Tradicionalmente se ha utilizado el hidróxido de calcio como material de elección para este tratamiento, sin embargo, sus efectos adversos han promovido el desarrollo y utilización de agregado trióxido mineral (MTA), del cual aún existe controversia sobre una mayor efectividad. Métodos: Realizamos una búsqueda en Epistemonikos, la mayor base de datos de revisiones sistemáticas en salud, la cual es mantenida mediante el cribado de múltiples fuentes de información, incluyendo MEDLINE, EMBASE, Cochrane, entre otras. Extrajimos los datos desde las revisiones identificadas, analizamos los datos de los estudios primarios, realizamos un metanálisis y preparamos una tabla de resumen de los resultados utilizando el método GRADE. Resultados y conclusiones: Identificamos cuatro revisiones sistemáticas que en conjunto incluyeron siete estudios primarios, de los cuales, cuatro corresponden a ensayos aleatorizados. Concluimos que el recubrimiento directo con agregado trióxido mineral (MTA) comparado con hidróxido de calcio probablemente aumenta el éxito clínico y que podría aumentar la sobrevida pulpar, pero la certeza de la evidencia es baja.
\end{abstract}

\section{PALABRAS CLAVE}

Recubrimiento directo; Dentición permanente; Caries; Agregado trióxido mineral; Hidróxido de calcio; Epistemonikos, GRADE.

\section{ABSTRACT}

Introduction: Direct pulp capping has been suggested as the treatment of exposed vital pulp. Conventionally calcium hydroxide $(\mathrm{CH})$ has been the main biomaterial option for maintaining pulp vitality, but its adverse effects have promoted the development and use of mineral trioxide aggregate (MTA). However, there is still uncertainty regarding its effectiveness. Methods: We searched in Epistemonikos, the largest database of systematic reviews in health, which is maintained by screening multiple information sources, including MEDLINE, EMBASE, Cochrane, among others. We extracted data from the systematic reviews, reanalyzed data of primary studies, conducted a metaanalysis and generated a summary of findings table using the GRADE approach. Results and conclusions: We identified four systematic reviews including seven studies overall, of which four were randomized trials. We conclude that direct pulp capping with mineral trioxide aggregate (MTA) probably improves clinical success rate and may improve pulp survival rate, however, the certainty of the evidence has been assessed as low.

Int. J. Inter. Dent Vol. 13(3); 181-185, 2020. 


\section{PROBLEMA}

El recubrimiento pulpar directo es un método para tratar la pulpa vital expuesta que consiste en la colocación de un material dental sobre el área afectada para facilitar la formación de dentina reparativa y mantener la vitalidad pulpar ${ }^{[1]}$. Tradicionalmente, el hidróxido de calcio ha sido considerado como gold standard para este tratamiento, pero su uso ha sido cuestionado en los últimos años debido a efectos adversos tales como disolución del material, formación de brechas y microfiltración bacteriana ${ }^{[1]}$.

Frente a ello, se ha potenciado el desarrollo de nuevos materiales para el recubrimiento pulpar directo, destacando el agregado trióxido mineral (MTA): un cemento hidráulico compuesto de cemento Portland tipo I, óxido de bismuto y sulfato de calcio dihidratado. Sus principales ventajas se asocian a la biocompatibilidad y bioactividad del material, que promueven la formación de tejido mineralizado uniforme y proporcionan una mejor capacidad de sellado y menor respuesta inflamatoria ${ }^{[1]}$

Sin embargo, el uso de agregado trióxido mineral (MTA) por sobre el hidróxido de calcio sigue siendo controvertido, ya que requiere de un largo tiempo de fraguado, es de difícil manipulación y potencialmente afectaría a la decoloración dentaria.

Este resumen busca evaluar la efectividad, seguridad y potenciales ventajas del uso de agregado trióxido mineral (MTA) en comparación con hidróxido de calcio como material para recubrimiento pulpar directo en dentición permanente con caries dentinaria profunda.

\section{MÉTODOS}

Realizamos una búsqueda en Epistemonikos, la mayor base de datos de revisiones sistemáticas en salud, la cual es mantenida mediante búsquedas en múltiples fuentes de información, incluyendo MEDLINE, EMBASE, Cochrane, entre otras. Extrajimos los datos desde las revisiones identificadas y analizamos los datos de los estudios primarios. Con esta información, generamos un resumen estructurado denominado FRISBEE (Friendly Summaries of Body of Evidence using Epistemonikos), siguiendo un formato preestablecido, que incluye mensajes clave, un resumen del conjunto de evidencia (presentado como matriz de evidencia en Epistemonikos), metanálisis del total de los estudios cuando sea posible, una tabla de resumen de resultados con el método GRADE y una sección de otras consideraciones para la toma de decisión.

\section{Mensajes clave}

- El recubrimiento directo con agregado trióxido mineral (MTA) comparado con hidróxido de calcio probablemente aumenta el éxito clínico.

- El recubrimiento directo con agregado trióxido mineral (MTA) comparado con hidróxido de calcio podría aumentar la sobrevida pulpar (certeza de la evidencia baja).

- No se encontró evidencia evaluando los desenlaces decoloración dentaria, dolor, pérdida de pieza dentaria o efectos adversos.
Acerca del conjunto de evidencia para esta pregunta

Encontramos cuatro revisiones sistemáticas [2], [3], [4], [5] que incluyeron siete estudios primarios ${ }^{[6],[7],[8],[9],[10],[11],}$

Cuál es la evidencia ${ }^{[12]}$ de los cuales, cuatro son ensayos

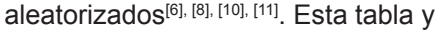

Véase matriz de evidencia en Epistemonikos más abajo. el resumen en general se basan en estos últimos, dado que los estudios observacionales no aumentaban la certeza de la evidencia existente, ni entregaban información adicional relevante.

Todos los ensayos incluyeron a pacientes con caries dentinaria profunda en dentición permanente.

Dos ensayos $^{[6],[11]}$, incluyeron pacientes con dentición permanente madura $e$ inmadura, con un rango etáreo entre los 8 y los 90 años de edad (edad promedio de 38 años).

Qué tipo de pacientes incluyeron los estudios*

Dos ensayos ${ }^{[8], ~[10] ~ i n c l u y e r o n ~ p a c i e n t e s ~}$ sólo con dentición permanente madura, mayores de 18 años.

Un ensayo ${ }^{[6]}$, incluyó pacientes en que la exposición pulpar en diente permanente se produjo de forma accidental, por trauma o provocada por caries.

Todos los ensayos compararon recubrimiento pulpar directo con

Qué tipo de intervenciones incluyeron los estudios* agregado trióxido mineral (MTA) contra tratamiento estándar con hidróxido de calcio.

Los ensayos reportaron múltiples desenlaces, los cuales fueron agrupados por las revisiones sistemáticas de la siguiente manera:

\section{- Éxito clínico}

- Sobrevida pulpar

Qué tipo de desenlaces

- Respuesta inflamatoria

midieron

- Formación de puente dentinario

El seguimiento de un ensayo ${ }^{[6]}$ fue de 12 meses. La información sobre el seguimiento del resto de los ensayos ${ }^{[8]}$

[10], [11] no fue presentada por las revisiones sistemáticas identificadas.

\section{RESUMEN DE LOS RESULTADOS}

La información sobre los efectos de agregado trióxido mineral (MTA) comparado con hidróxido de calcio como material de recubrimiento directo en dentición permanente con caries dentinaria profunda está basada en cuatro ensayos aleatorizados ${ }^{[6],[8],[10], ~[11]}$ que incluyeron 623 pacientes.

Un ensayo midió el desenlace éxito clínico (376 pacientes) ${ }^{[6]}$. Cuatro ensayos midieron el desenlace sobrevida pulpar (623 pacientes) [6], [8], [10], [11], pero ninguna revisión permitió la extracción de datos de manera que pudieran ser incorporados a un metanálisis, por lo que la información de este desenlace se presenta como síntesis narrativa.

El resumen de los resultados es el siguiente:

- El recubrimiento directo con agregado trióxido mineral (MTA) comparado con hidróxido de calcio probablemente aumenta el éxito clínico (certeza de la evidencia moderada).

- El recubrimiento directo con agregado trióxido mineral (MTA) comparado con hidróxido de calcio podría aumentar la sobrevida pulpar (certeza de la evidencia baja).

- No se encontró evidencia evaluando el desenlace decoloración dentaria.

- No se encontró evidencia evaluando el desenlace dolor.

- No se encontró evidencia evaluando el desenlace pérdida de pieza dentaria.

- No se encontró evidencia evaluando el desenlace efectos adversos asociados al tratamiento. 


\section{Recubrimiento directo con agregado trióxido mineral (MTA) o hidróxido de calcio para dentición permanente con caries dentinaria profunda}

\section{Pacientes Dentición permanente con caries dentinaria profunda \\ Intervención Recubrimiento directo con agregado trióxido mineral (MTA) \\ Comparación Recubrimiento directo con hidróxido de calcio}

\begin{tabular}{|c|c|c|c|c|}
\hline \multirow{3}{*}{ Desenlaces } & \multicolumn{2}{|c|}{ Efecto absoluto $*$} & \multirow{3}{*}{$\begin{array}{l}\text { Efecto } \\
\text { relativo } \\
(\text { IC } 95 \%)\end{array}$} & \multirow{3}{*}{$\begin{array}{l}\text { Certeza de } \\
\text { la evidencia } \\
\text { (GRADE) }\end{array}$} \\
\hline & $\begin{array}{c}\text { CON } \\
\text { hidróxido de calcio }\end{array}$ & $\begin{array}{c}\text { CON } \\
\text { agregado trióxido } \\
\text { mineral (MTA) }\end{array}$ & & \\
\hline & \multicolumn{2}{|c|}{ Diferencia: pacientes por 1000} & & \\
\hline \multirow[b]{2}{*}{ Éxito clínico** } & 743 por 1000 & 862 por 1000 & \multirow{2}{*}{$\begin{array}{l}\operatorname{RR} 1,16 \\
(1,05 \text { a } \\
1,29)\end{array}$} & \multirow{2}{*}{$\begin{array}{c}\oplus \oplus \oplus \bigcirc^{1} \\
\text { Moderada }\end{array}$} \\
\hline & \multicolumn{2}{|c|}{$\begin{array}{l}\text { Diferencia: } 119 \text { pacientes más } \\
\text { (Margen de error: } 37 \text { a } 215 \text { más) }\end{array}$} & & \\
\hline $\begin{array}{l}\text { Sobrevida } \\
\text { pulpar*** }\end{array}$ & \multicolumn{3}{|c|}{$\begin{array}{c}\text { Una revisión sistemática [4] que incluyó tres ensayos [6], } \\
\text { [8], [10] reportaron que la sobrevida pulpar fue alta en el } \\
\text { grupo que recibió agregado trióxido mineral (MTA) y } \\
\text { moderada en el grupo que recibió hidróxido de calcio } \\
\text { Sólo un ensayo [11] reportó que la sobrevida pulpar fue } \\
\text { alta en ambos grupos. }\end{array}$} & $\begin{array}{c}\oplus \oplus \bigcirc \bigcirc^{1,2} \\
\text { Baja }\end{array}$ \\
\hline $\begin{array}{l}\text { Decoloración } \\
\text { dentaria }\end{array}$ & \multicolumn{2}{|c|}{$\begin{array}{c}\text { El desenlace decoloración dentaria no fue } \\
\text { medido o reportado. }\end{array}$} & -- & -- \\
\hline Dolor & \multicolumn{2}{|c|}{ El desenlace dolor no fue medido o reportado. } & -- & -- \\
\hline $\begin{array}{l}\text { Pérdida de } \\
\text { pieza dentaria }\end{array}$ & \multicolumn{2}{|c|}{$\begin{array}{l}\text { El desenlace pérdida de pieza dentaria no fue } \\
\text { medido o reportado. }\end{array}$} & -- & -- \\
\hline $\begin{array}{l}\text { Efectos } \\
\text { adversos }\end{array}$ & \multicolumn{2}{|c|}{$\begin{array}{l}\text { El desenlace efectos adversos asociados al } \\
\text { tratamiento no fue medido o reportado. }\end{array}$} & -- & -- \\
\hline
\end{tabular}

Margen de error: Intervalo de confianza del 95\% (IC 95\%).

RR: Riesgo relativo.

GRADE: Grados de evidencia del GRADE Working Group (ver más adelante).

*Los riesgos CON hidróxido de calcio están basados en los riesgos del grupo control en los estudios. El riesgo CON agregado trióxido mineral (MTA) (y su margen de error) está calculado a partir del efecto relativo (y su margen de error).

**Éxito clínico medido como ausencia de movilidad patológica, ausencia de fístula, ausencia de sensibilidad a la palpación, ausencia de dolor espontáneo post tratamiento y ausencia de patología radiográfica.

***Sobrevida pulpar medida a los 6 - 12 meses posterior a la intervención. Se considera sobrevida pulpar baja cuando es menor al $40 \%$, moderada entre el $40 \%$ y $80 \%$ y alta cuando es mayor al $80 \%$.

${ }^{1}$ Se disminuyó un nivel de certeza de la evidencia por riesgo de sesgo, ya que los participantes y evaluadores de los desenlaces del ensayo no fueron ciegos.

2 Se disminuyó un nivel de certeza de la evidencia por inconsistencia, dado que los diferentes ensayos llegaron a conclusiones distintas. 


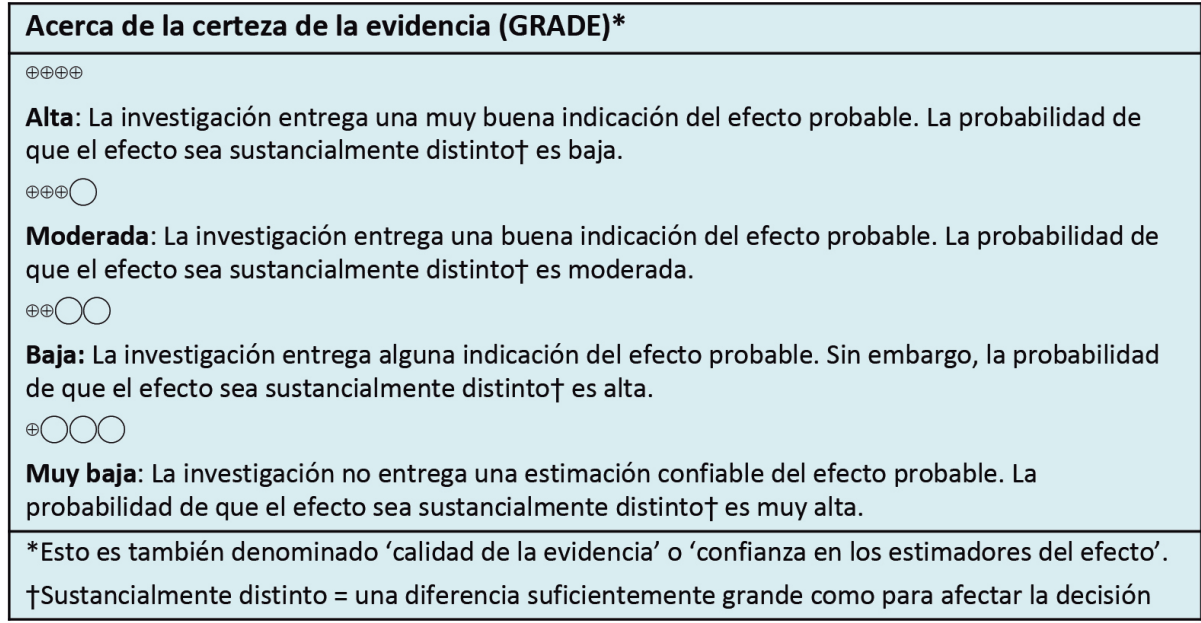

\section{OTRAS CONSIDERACIONES PARA LA TOMA DE DECISIÓN}

\section{A quién se aplica y a quién no se aplica esta evidencia}

- Los resultados de este resumen son aplicables a todos los pacientes con caries dentinaria profunda en dientes permanentes maduros o inmaduros con un diagnóstico pulpar de pulpa normal o pulpitis reversible.

- Si bien los ensayos no incluyeron a pacientes menores de 7 años, no existirían razones clínicas para no aplicar los resultados a esta población, siempre y cuando se trate de caries profundas en dentición permanente con un diagnóstico pulpar de pulpa normal o pulpitis reversible.

- Esta evidencia no aplica a pacientes con dentición primaria.

\section{Sobre los desenlaces incluidos en este resumen}

- Los desenlaces seleccionados son aquellos considerados críticos para la toma de decisión de acuerdo a la opinión de los autores de este resumen, de los cuáles sólo los desenlaces éxito clínico y sobrevida pulpar coinciden con los evaluados por las principales revisiones sistemáticas incluidas.

- Los desenlaces decoloración dentaria, dolor, pérdida de pieza dentaria y efectos adversos no fueron reportados por las revisiones evaluadas. Sin embargo, son considerados importantes, ya que afectan a la calidad de vida del paciente.

- El desenlace "éxito clínico", es crítico para la toma de decisiones en salud, ya que supone un tratamiento definitivo para el paciente de forma efectiva, implicando la ausencia de movilidad patológica, fístula, sensibilidad a la palpación, dolor espontáneo post tratamiento y patología radiográfica.

- El desenlace "sobrevida pulpar", también es crítico para la toma de decisiones en salud, ya que implica la conservación de la vitalidad pulpar.

\section{Balance daño/beneficio y certeza de la evidencia}

- La evidencia muestra un posible beneficio en el éxito clínico, mostrando ventajas al utilizar agregado trióxido mineral (MTA) comparado con hidróxido de calcio para el recubrimiento directo de caries dentinaria profunda en dientes permanentes. Sin embargo, no existe evidencia que mida el posible riesgo de manifestar dolor, decoloración dentaria, pérdida de la pieza dentaria o efectos adversos de la intervención.

- Por otro parte, la duración del seguimiento sólo está disponible para uno de los ensayos incluidos (12 meses) ${ }^{[6]}$, lo que dificulta la proyección del éxito clínico y sobrevida pulpar a largo plazo de ambas intervenciones.

- No es posible hacer un adecuado balance entre riesgos y beneficios del recubrimiento pulpar directo con agregado trióxido mineral (MTA) debido a la incertidumbre existente sobre sus riesgos, asociada a las limitaciones de la evidencia existente.

\section{Consideraciones de recursos}

- Ninguno de los estudios incluidos realizó un análisis de costos entre el recubrimiento directo con agregado trióxido mineral (MTA) y el recubrimiento directo con hidróxido de calcio. Sin embargo, existe evidencia científica disponible al respecto.

- Un estudio en Alemania[13] evaluó la relación costo-efectividad del agregado trióxido mineral (MTA) versus el hidróxido de calcio como material para recubrimiento pulpar directo, concluyendo que agregado trióxido mineral (MTA) es más costo efectivo, pese a representar un mayor costo inicial, ya que se evitan costos asociados a intervenciones posteriores más invasivas o re tratamientos.

- Un estudio en Australia ${ }^{[14]}$ evaluó la educación sobre el uso de agregado trióxido mineral (MTA) entre los miembros de la sociedad australiana de endodoncia, concluyendo que la experiencia en el manejo de agregado trióxido mineral (MTA) como material es una barrera más grande para su uso generalizado que su costo.

- El costo económico del agregado trióxido mineral (MTA) como material para recubrimiento pulpar directo podría reducirse colocando el cemento no utilizado en un recipiente hermético, proporcionando suficiente material para completar otros tres o cinco procedimientos diferentes a partir de un solo paquete abierto[15]

- Finalmente, los dientes que reciben tratamiento de conducto radicular son más propensos a fallas debido a fracturas, microinfiltración y la aparición o recurrencia de patología periapical. Por ende, la preservación de la vitalidad pulpar no debe ser vista como una alternativa de tratamiento costosa, sino más bien como una estrategia confiable para prevenir o posponer procedimientos dentales más invasivos ${ }^{[15]}$.

\section{Qué piensan los pacientes y sus tratantes}

- Actualmente, la mayoría de los profesionales de la salud se inclinan a favor del uso de agregado trióxido mineral (MTA) por sobre el hidróxido de calcio para recubrimiento directo en dientes permanentes con caries dentinaria profunda.

- Esta preferencia entre los endodoncistas se debe principalmente a las características superiores del agregado trióxido mineral (MTA) como material para recubrimiento pulpar directo en comparación al hidróxido de calcio, entre ellas: biocompatibilidad, sellado hermético que evita la microfiltración, bioactividad al inducir la formación de puentes dentinarios de alta calidad (buen espesor y baja porosidad), mayor resistencia mecánica y menor inflamación a nivel pulpar. Si bien, se deben tener ciertas consideraciones para su uso como: manipulación que requiere de entrenamiento previo, mayor tiempo de fraguado, posibilidad de provocar tinciones en dientes anteriores y costo más elevado, sus propiedades físicas, biológicas y mecánicas constituyen un tratamiento que se considera menos invasivo en comparación al hidróxido de calcio[16].

- Además, es importante considerar la adhesión que tendrá el material utilizado con los materiales restauradores definitivos en la toma de decisión clínica. Las bases cavitarias y/o la resina compuesta no se adhieren al hidróxido de calcio. En el caso del agregado trióxido mineral (MTA) tampoco existe adhesión química, pero el sellado que se logra es hermético. Esto podría considerarse una ventaja en pacientes con alto riesgo de pérdida de las restauraciones como es el caso de la población pediátrica.

- Finalmente, a pesar de que la evidencia analizada sólo respalda el uso de la intervención desde su éxito clínico y que no se tiene certeza sobre posibles efectos adversos, es probable que los tratantes no alteren sus preferencias. Sin embargo, es importante considerar otros aspectos en la toma de decisión, como los valores y preferencias del paciente.

\section{Diferencias entre este resumen y otras fuentes}

- Las conclusiones de este resumen concuerdan con dos de las cuatro

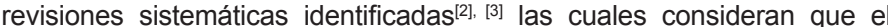
recubrimiento pulpar directo con agregado trióxido mineral (MTA) muestra un mayor éxito clínico que el hidróxido de calcio.

- Las otras dos revisiones ${ }^{[4],[5]}$ concluyen que no existirían diferencias en el uso de agregado trióxido mineral (MTA) como material para recubrimiento pulpar directo en comparación con el hidróxido de calcio. Sin embargo, estas revisiones no cuentan con un número suficiente de ensayos aleatorizados a largo plazo que aporten datos para realizar una 
conclusión adecuada sobre el uso de un material específico ${ }^{[4],[5]}$

- La Guide to Clinical Endodontics de la American Association of Endodontists ${ }^{[17]}$, la Quality guidelines for endodontic treatment: consensus report of the European Society of Endodontology $y^{[18]}$ y la adaptación de guías clínicas de la asociación americana de endodoncia según la sociedad de endodoncia de Chile ${ }^{[19]}$ recomiendan el uso de agregado trióxido mineral (MTA) por sobre hidróxido de calcio para el recubrimiento pulpar directo en diente permanente con caries dentinaria profunda y diagnóstico de pulpa normal o pulpitis reversible.

\section{¿Puede que cambie esta información en el futuro?}

- La probabilidad de que las conclusiones de este resumen cambien frente a nueva evidencia es baja respecto al éxito clínico y alta para el resto de los desenlaces evaluados, considerando la incertidumbre de la evidencia existente.

- Se identificó un ensayo aleatorizado en curso $^{[20]}$ en la International Clinical Trials Registry Platform de la Organización Mundial de la Salud que podría arrojar datos relevantes para el desenlace de éxito clínico y sobrevida pulpar.

- Se identificaron tres revisiones sistemáticas en curso [21], [22], [23] en el International prospective register of systematic reviews (PROSPERO) del National Institute for Health Research que podrían arrojar datos relevantes para el desenlace de éxito clínico.

\section{CÓMO REALIZAMOS ESTE RESUMEN}

Mediante métodos automatizados y colaborativos recopilamos toda la evidencia relevante para la pregunta de interés y la presentamos en una matriz de evidencia.

\section{Siga el enlace para acceder a la versión interactiva:} Recubrimiento directo con agregado trióxido mineral (MTA) comparado con hidróxido de calcio en diente permanente con caries dentinaria profunda

\section{NOTAS}

Si con posterioridad a la publicación de este resumen se publican nuevas revisiones sistemáticas sobre este tema, en la parte superior de la matriz se mostrará un aviso de "nueva evidencia".

Este artículo es parte del proyecto síntesis de evidencia de Epistemonikos. Se elabora con una metodología preestablecida, siguiendo rigurosos estándares metodológicos y proceso de revisión por pares interno. Cada uno de estos artículos corresponde a un resumen, denominado FRISBEE (Friendly Summary of Body of Evidence using Epistemonikos), cuyo principal objetivo es sintetizar el conjunto de evidencia de una pregunta específica, en un formato amigable a los profesionales clínicos. Sus principales recursos se basan en la matriz de evidencia de Epistemonikos y análisis de resultados usando metodología GRADE. Mayores detalles de los métodos para elaborar este FRISBEE están descritos aquí (http://dx.doi.org/10.5867/medwave.2014.06.5997)

La Fundación Epistemonikos es una organización que busca acercar la información a quienes toman decisiones en salud, mediante el uso de tecnologías. Su principal desarrollo es la base de datos Epistemonikos (www.epistemonikos.org).

\section{DECLARACIÓN DE CONFLICTOS DE INTERESES}

Los autores declaran no tener conflictos de intereses con la materia de este artículo.

\section{AGRADECIMIENTOS}

Este resumen de evidencia fue elaborado con el apoyo metodológico del Centro Evidencia UC, Facultad de Medicina, Pontificia Universidad Católica de Chile.

\section{Bibliografía}

1. Komabayashi T, Zhu Q, Eberhart R, Imai Y. Current status of direct pulp-capping materials for permanent teeth. Dent Mater J. 2016;35(1):1-12. doi: 10.4012/dmj.2015013. PMID: 26830819

2. Aguilar $P$, Linsuwanont $P$. Vital pulp therapy in vital permanent teeth with cariously exposed pulp: a systematic review. J Endod. 2011 May;37(5):581-7. doi: 10.1016/j. joen.2010.12.004. Epub 2011 Mar 5. PMID: 21496652.

3. Li Z, Cao L, Fan M, Xu Q. Direct Pulp Capping with Calcium Hydroxide or Mineral Trioxide Aggregate: A Meta-analysis. J Endod. 2015 Sep;41(9):1412-7. doi: 10.1016/j. joen.2015.04.012. Epub 2015 May 16. PMID: 25990198.

4. Munir A, Zehnder M, Rechenberg DK. Wound Lavage in Studies on Vital Pulp Therapy of Permanent Teeth with Carious Exposures: A Qualitative Systematic Review. J Clin Med. 2020 Apr 1;9(4):984. doi: 10.3390/jcm9040984. PMID: 32244782; PMCID: PMC7231275.

5. Schwendicke F, Brouwer F, Schwendicke A, Paris S. Different materials for direct pulp capping: systematic review and meta-analysis and trial sequential analysis. Clin Ora Investig. 2016 Jul;20(6):1121-32. doi: 10.1007/s00784-016-1802-7. Epub 2016 Apr 2. PMID: 27037567.

6. Hilton TJ, Ferracane JL, Mancl L; Northwest Practice-based Research Collaborative in Evidence-based Dentistry (NWP). Comparison of $\mathrm{CaOH}$ with MTA for direct pulp capping: a PBRN randomized clinical trial. J Dent Res. 2013 Jul;92(7 Suppl):16S 22S. doi: 10.1177/0022034513484336. Epub 2013 May 20. PMID: 23690353; PMCID: PMC3706175.

7. Mente J, Geletneky B, Ohle M, Koch MJ, Friedrich Ding PG, Wolff D, Dreyhaupt J, Martin N, Staehle HJ, Pfefferle T. Mineral trioxide aggregate or calcium hydroxide direct pulp capping: an analysis of the clinical treatment outcome. J Endod. 2010 May;36(5):806-13. doi: 10.1016/j.joen.2010.02.024. PMID: 20416424.

8. Kundzina R, Stangvaltaite L, Eriksen HM, Kerosuo E. Capping carious exposures in adults: a randomized controlled trial investigating mineral trioxide aggregate versus calcium hydroxide. Int Endod J. 2017 Oct;50(10):924-932. doi: 10.1111/iej.12719. Epub 2016 Nov 28. PMID: 27891629.

9. Mente J, Hufnagel S, Leo M, Michel A, Gehrig H, Panagidis D, Saure D, Pfefferle T. Treatment outcome of mineral trioxide aggregate or calcium hydroxide direct pulp capping: long-term results. J Endod. 2014 Nov;40(11):1746-51. doi: 10.1016/j. joen.2014.07.019. Epub 2014 Sep 13. PMID: 25227216

10. Suhag K, Duhan J, Tewari S, Sangwan P. Success of Direct Pulp Capping Using Mineral Trioxide Aggregate and Calcium Hydroxide in Mature Permanent Molars with Pulps Exposed during Carious Tissue Removal: 1-year Follow-up. J Endod. 2019 Jul;45(7):840-847. doi: 10.1016/j.joen.2019.02.025. Epub 2019 May 16. PMID: 31104819.

11. Brizuela C, Ormeño A, Cabrera C, Cabezas R, Silva Cl, Ramírez V, Mercade M. Direct Pulp Capping with Calcium Hydroxide, Mineral Trioxide Aggregate, and Biodentine in Permanent Young Teeth with Caries: A Randomized Clinical Trial. J Endod. 2017 Nov;43(11):1776-1780. doi: 10.1016/j.joen.2017.06.031. Epub 2017 Sep 14. PMID: 28917577
12. Cho SY, Seo DG, Lee SJ, Lee J, Lee SJ, Jung IY. Prognostic factors for clinical outcomes according to time after direct pulp capping. J Endod. 2013 Mar;39(3):327-31. doi: 10.1016/j.joen.2012.11.034. Epub 2013 Jan 16. PMID: 23402502

13. Schwendicke F, Brouwer F, Stolpe M. Calcium Hydroxide versus Mineral Trioxide Aggregate for Direct Pulp Capping: A Cost-effectiveness Analysis. J Endod. 2015 Dec;41(12):1969-74. doi: 10.1016/j.joen.2015.08.019. Epub 2015 Oct 2. PMID: 26435470.

14. Ha WN, Duckmanton P, Kahler B, Walsh LJ. A survey of various endodontic procedures related to mineral trioxide aggregate usage by members of the Australian Society of Endodontology. Aust Endod J. 2016 Dec;42(3):132-138. doi: 10.1111/ aej.12170. Epub 2016 Sep 15. PMID: 27628614.

15. Bogen G, Hilton T. Do the Benefits of MTA for Direct Pulp Capping Justify the Cost? Inside Dentistry [Internet]. May 2014. [cited 2020 Jul 5]; 10(5): 30. Available from: https://www.aegisdentalnetwork.com/id/2014/05/do-the-benefits-of-mta-for-direct-pulpcapping-justify-the-cost

16. Friedlander L, McElroy K, Daniel B, Cullinan M, Hanlin S. Direct pulp capping of permanent teeth in New Zealand general dental practice--a practice based research study. N Z Dent J. 2015 Jun;111(2):58-64. PMID: 26219182.

17. American Association of Endodontists. Guide to Clinical Endodontics. AAE Guid. 2013;88(7):8-9.

18. European Society of Endodontology. Quality guidelines for endodontic treatment: consensus report of the European Society of Endodontology. Int Endod J. 2006 Dec;39(12):921-30. doi: 10.1111/j.1365-2591.2006.01180.x. PMID: 17180780.

19. Antúnez M, Araya P, Dezerega A, Garrido M, Ljubetic O, Navia M, Olguín C, Pelegr M, Soto M, Viñuela V, Valenzuela W. Adaptación Guías clínicas AAE. Soc Endod Chile. 2014;5:19.

20. Mahapratra J. Comparative evaluation of the efficacy of Theracal LC, Mineral Trioxide Aggregate and Biodentine as Direct Pulp Capping Materials in patients with pulpal exposure in posterior teeth. CTRI 2020 023047. Available from: https://apps.who. int/trialsearch/Trial2.aspx?TriallD=CTRI/2020/01/023047

21. Fasoulas A, Keratiotis G, Augustus M, Pandis N, Spineli L. Comparative efficacy of materials used in patients undergoing pulpotomy or direct pulp capping: A systematic review with network meta-analysis. PROSPERO 2020 CRD42020127239 Available from: https://www.crd york.ac.uk/prospero/display record php?ID=CRD42020127239 22. Shah S, Singh S, Podar R, Chandrasekhar P, Pradhan J, Jain R, Kumar V. Comparative Evaluation Of Success Rate Of Calcium Silicate Based Cements and Calcium Hydroxide In Vital Pulp Therapy: A Systematic Review. PROSPERO 2020 CRD42020184786 Available from: https://www.crd.york.ac.uk/prospero/display_record. php?ID=CRD42020184786

23. Tamayo M, Hincapie S, Chambrone L, Ochoa P. New materials for direct pulp capping in temporary and permanent young teeth: a systematic review. PROSPERO 2019 CRD42019135758 Available from: https://www.crd.york.ac.uk/prospero/display record.php?ID=CRD42019135758 